\title{
Neuro-0phthalmic Syndromes and Processing Speed in Multiple Sclerosis
}

\author{
Silvana L. Costa, PhD, Óscar F. Gonçalves, PhD, Nancy D. Chiaravalloti, PhD, \\ John DeLuca, PhD, Jorge Almeida, PhD
}

\begin{abstract}
The impact of prior neuro-ophthalmic syndromes on the performance on vision-based neuropsychological tasks in patients with multiple sclerosis (MS) is unknown. Two groups of MS participants, one with (Msos+) and the other without (Msos-), a history of neuro-ophthalmic syndromes, underwent neuropsychological assessment and were compared with healthy age- and education-matched controls (HC). Participants with Msos+ performed significantly worse on the symbol digit modalities test than the Msos $-(P<0.03)$ and the $\mathrm{HC}$ groups $(P<0.01)$ and coding $(P<0.01)$. A clinical history of neuro-ophthalmic syndromes is associated with reduced performance on visual processing speed tasks.
\end{abstract}

Journal of Neuro-0phthalmology 2015;0:1-6

doi: 10.1097/WNO.0000000000000272

○ 2015 by North American Neuro-Ophthalmology Society

$\mathbf{I}^{\mathrm{n}}$ n patients with multiple sclerosis (MS), acute episodes of neuro-ophthalmological syndromes are common, appearing both as a first symptom and during the disease

Neuropsychophysiology Lab-CIPsi (SLC, OFG), University of Minho, Braga, Portugal; Neuropsychology and Neuroscience Research (SLC, NDC, JDL), Kessler Foundation, West Orange, New Jersey; Department of Physical Medicine and Rehabilitation (SLC, OFG, NDC), Rutgers New Jersey Medical School, Newark, New Jersey; Department of Counseling and Applied Educational Psychology(OFG), Bouvé College of Health Sciences, Northeastern University, Boston, Massachusetts; Spaulding Neuromodulation Center (OFG), Department of Physical Medicine and Rehabilitation, Spaulding Rehabilitation Hospital, Harvard Medical School, Boston, Massachusetts; Department of Neurology (OFG), Rutgers New Jersey Medical School, Newark, New Jersey; Faculty of Psychology and Educational Sciences (JA), University of Coimbra, Coimbra, Portugal; Proaction Laboratory (JA), Faculty of Phycology and Educational Sciences, University of Coimbra, Portugal

Supported partially by a $\mathrm{PhD}$ fellowship from Fundação para a Ciência e Tecnologia Portuguesa (Foundation for Science and Technology of Portugal, fellowship number SFRH/BD/45591/2008) to S. L. Costa. J. Almeida was supported by Foundation for Science and Technology of Portugal, project grant PTDC/MHC PCN/3575/ 2012, and programa COMPETE.

The authors report no conflicts of interest.

Address correspondence to Silvana L. Costa, PhD, Kessler Foundation, 300 Executive Drive, Suite 70, West Orange, NJ 07052; E-mail: scosta@kesslerfoundation.org course (1). This involvement may affect the afferent and efferent visual pathways. Although full clinical recovery from an acute episode is often achieved, long-term abnormalities may persist (2). For example, a longitudinal study performed by Raz et al (3) showed that after an acute episode of optic neuritis, although visual evoked potential (VEP) amplitudes and static visual functions may recover within 4 months, VEP latency and motion perception may remain significantly impaired beyond 12 months. Tilikete et al (4) demonstrated that, in participants with MS, ocular motor problems are associated with decreased visual acuity and visual functioning scores (5). Cognitive impairment also is common in MS affecting between $43 \%$ and $70 \%$ of participants. Abnormalities in attention, information processing speed (PS), memory, executive functions, and visuoperceptual skills frequently are present (4). Despite the fact that both visual abnormalities and cognitive impairment are common in MS, the complex interaction between the 2 is not completely understood. The few studies that have addressed this relationship found that mild deficits in visual acuity (6-8) and abnormalities in cognitive processing in the ocular motor system (9) were associated with poorer cognitive performance. However, these studies did not report neuro-ophthalmic examination results in these participants nor did they examine the influence of these findings on cognition.

The aim of our study was to determine whether a history of neuro-ophthalmic syndromes might be related to poor performance on neuropsychological tasks in participants with relapse-remitting MS with normal visual acuity.

\section{METHODS}

\section{Participants}

Eighteen participants diagnosed with relapsing-remitting MS (10) and 9 healthy controls participated in this study. Before enrollment, all participants signed an informed 
consent approved by the Institutional Review Board of the Kessler Foundation.

Participants were excluded if they reported any history of medical or psychiatric disorders that could substantially influence cognitive function. This included, but was not limited to, traumatic brain injury, alcohol or drug dependence (past or present), learning disability, bipolar disorder, schizophrenia, or stroke. Participants were excluded if they reported any visual problems at time of testing. Participants with MS must not have experienced an exacerbation of symptoms in at least 4 weeks and had not taken corticosteroids, benzodiazepines, or neuroleptics.

Participants with MS were divided into 2 groups according to their self-reported history of MS-related neuro-ophthalmic syndromes. One group included MS participants with a history of at least 1 episode of neuroophthalmic syndromes such as nystagmus or optic neuritis (MSos+ $[\mathrm{N}=12]$ ). The second group consisted of participants with MS who did not have any history of neuroophthalmic syndromes, (MSos- $[\mathrm{N}=6])$. Participants in the HC group did not report any history of visual disturbances. The 3 groups did not differ significantly in terms of age $\left(F_{2,24}=0.61, \mathrm{NS}\right)$, years of education $\left(F_{2,24}=1.08\right.$, NS) or overall intelligence as measured with WRAT-III word reading subtest $\left(F_{2,24}=2.48\right.$, NS) (Table 1$)$. The $2 \mathrm{MS}$ groups also did not differ on the number of months since diagnosis of MS $\left(t_{16}=1.44, \mathrm{NS}\right)$ or months since last exacerbation $\left(t_{16}=-0.91, \mathrm{NS}\right)$. As such, the 2 groups seem to present similar disease progression.

The Snellen high contrast vision chart was used to measure best-corrected binocular visual acuity. A binocular Snellen acuity score of less than 20/70 in both eyes with optical correction was established as the cutoff point for study enrollment because this score has been regarded as the minimum visual acuity necessary for neuropsychological testing (11). An analysis of variance (ANOVA) with the
Snellen acuity test scores revealed no significant differences between the 3 groups for acuities in both eyes (right eye $F_{2,23}=0.46$, NS; left eye $F_{2,23}=1.02$, NS) (Table 1).

\section{Neuropsychological Assessment}

All participants were administered the Minimal Neuropsychological Assessment of Multiple Sclerosis (MACFIMS; for details, see Benedict et al (12)). MACFIMS is a comprehensive battery composed of 7 tests measuring verbal fluency (Delis-Kaplan Executive Functions System [DKEFS] Verbal Fluency Test (13)), visual perception (Judgment of Line Orientation Test [JLO] (14)), verbal and visuospatial learning and memory (California Verbal Learning Test II [CVLT II] (15), and the Brief Visuospatial Memory Test Revised [BVMT-R] (16)), PS (Symbol Digit Modalities Test [SDMT] (17)), working memory (WM)/PS (Paced Auditory Serial Addition Test [PASAT] (18)), and executive functions (DKEFS Sorting Test (13)). For all the tests performances, z scores values were calculated based on the published norms proposed (19).

Letter Number Sequencing (LNS), Arithmetic, and Digit Span (DS) subtests were used to assess WM (20), and the Coding (CO) and Symbol Search (SS) subtests were used to assess visual-motor PS (21).

Six of the tasks included in this comprehensive neuropsychological test battery are vision based; they use visual stimuli as their testing stimuli (JLO, SDMT, DKEFS Sorting, BVMT-R, SS, and CO). The 6 remaining tasks are auditory based because they are performed using auditory stimuli (DKEFS fluency, CVLT II, DS, PASAT, LNS, and Arithmetic). Additionally, from these 12 tests, seven have to be completed under a time constraint (SDMT, DKEFS sorting, and DKEFS fluency). For the remaining 5 tasks, participants had unlimited time to perform the task (JLO, BVMT-R, CVLT II, DS, and LNS).

TABLE 1. Demographic information of study participants

\begin{tabular}{lcccc}
\hline & $\begin{array}{c}\text { MS MSos }(\mathrm{N}=12) \\
\text { Mean }(\mathrm{SEM})\end{array}$ & $\begin{array}{c}\text { MS MSos }-(\mathrm{N}=6) \\
\text { Mean }(\mathrm{SEM})\end{array}$ & $\begin{array}{c}\mathrm{HC}(\mathrm{N}=9) \\
\text { Mean }(\mathrm{SEM})\end{array}$ & $F$ Score \\
\hline Age & $41.75(3.2)$ & $43.67(2.8)$ & $38.56(2.46)$ & $F_{2.24}=0.61$ \\
Years of education & $14.92(0.71)$ & $14.17(0.65)$ & $15.78(0.62)$ & $F_{2,24}=1.08$ \\
BDI total & $11.45(2.66)$ & $9.83(3.12)$ & $1.67(0.78)$ & $F_{2,23}=5.25 *$ \\
Acuity right eye & $20 / 31.67(3.81)$ & $20 / 29.17(4.3)$ & $20 / 26.88(2.3)$ & $F_{2,23}=0.46$ \\
Acuity left eye & $20 / 44.17(14.39)$ & $20 / 28.33(4.59)$ & $20 / 22.5(1.89)$ & $F_{2,23}=1.02$ \\
WRAT-III & $100.33(4.57)$ & $100(4.38)$ & $111.78(2.77)$ & $F_{2,24}=2.48$ \\
Months since diagnosis & $150.75(30.07)$ & $85.33(21.39)$ & - & $t_{16}=1.44$ \\
\hline
\end{tabular}

Acuity right eye and left eye represent the scores obtained by each participant on the Snellen high-contrast vision chart for the right and left eye.

${ }^{*} P<0.01$

BDI total, total score of the Beck Depression Inventory; MSos+, multiple sclerosis group with history of neuro-ophthalmic syndromes; MSos-, multiple sclerosis group without history of neuro-ophthalmic syndromes; SEM, standard error of mean. 


\section{Data Analyses}

Differences between groups on neuropsychological data were analyzed using ANOVA. Post hoc comparisons of the mean values were performed with Bonferroni correction when the ANOVAs revealed significant effects. Data are presented in terms of mean and standard error of mean (SEM). The criterion for statistical significance was established at $P<0.05$. All statistical analyses were performed using IBM SPSS Statistics for Mac OS (version 20).

\section{RESULTS}

\section{Vision-Based Neuropsychological Tasks}

Vision-based neuropsychological data are illustrated in Figure 1. Significant group differences were found for all PS tasks (SDMT $\left[F_{2,24}=7.37, P<0.01\right]$, SS $\left[F_{2,24}=8.26\right.$, $P<0.01]$; Coding $\left.\left[F_{2,24}=5.00, P<0.02\right]\right)$. A main effect of group was also found for the long-term visual memory task, BVMT-R Delay $\left(F_{2,24}=3.84, P<0.05\right)$.

On the SDMT, the MS group MSos+ demonstrated significantly worse performance $($ mean $=-1.97, \mathrm{SEM}=$ 0.43 ) compared with the MSos-group (mean $=-0.35$, $\mathrm{SEM}=0.29 ; P<0.04)$, and the $\mathrm{HC}$ group (mean $=$ $-0.07, \mathrm{SEM}=0.35 ; P<0.01)$. No significant difference was found between the MSos- and the HC groups.

On SS, a significant difference was noted on SS between the MSos+ group and the HC group (mean $=1.30, \mathrm{SEM}=$ 0.26 for HC group; $P<0.01)$ and between the MSosand the HC group $(P<0.04)$. Additionally, no significant difference was noted between MS groups (mean $=-0.31$, $\mathrm{SEM}=0.30$, for MSos+ group; and mean $=-0.06, \mathrm{SEM}=$ 0.36, for MSos-).

On coding, there was no significant difference between the MSos+ and MSos - groups (mean $=-0.67, \mathrm{SEM}=$ 0.40 , for MSos+ group and mean $=0.17, \mathrm{SEM}=0.49$, for MSos-) or the MSos- group and the $\mathrm{HC}$ group.
However, significantly poorer performance was observed for the MSos+ when compared with the $\mathrm{HC}$ group (mean = $0.96, \mathrm{SEM}=0.26 ; P<0.02)$.

Our measure of visual memory, the BVMT-R Delay showed no significant difference between the MSos+ $($ mean $=-1.57$, SEM $=0.43)$ and the MSos - groups $($ mean $=-1.21, \mathrm{SEM}=0.65)$ or the MSos - group and the HC group. However, the MSos+ group evidenced significantly poorer performance than the HC group $(P<0.04)$.

No differences between groups were found for JLO $\left(F_{2,24}=\right.$ $0.11, \mathrm{NS})$ or the DKEFS Sorting test $\left(F_{2,24}=0.57, \mathrm{NS}\right)$.

\section{Auditory-Based Neuropsychological Tasks}

Auditory-based neuropsychological tests are illustrated in Figure 2. A significant main effect of group for LNS $\left(F_{2,24}=\right.$ 8.76, $P<0.002)$ and DS $\left(F_{2,24}=3.68, P<0.05\right)$ was found. On LNS, the $2 \mathrm{MS}$ groups perform similarly (MSos+: mean $=-0.22, \mathrm{SEM}=0.18$; MSos $-:$ mean $=0.06, \mathrm{SEM}=$ $0.22)$. However, both the MSost and MSos- groups performed significantly worse on the LNS than the HC group (HC: mean $=1.04, \mathrm{SEM}=0.29$; MSos+; $P<0.01$ : MSos - ; $P<0.04)$.

A similar pattern of results was noted on DS with no significant difference between the 2 MS groups (MSost: mean $=-0.14$, SEM $=0.26 ;$ MSos $^{-}:$mean $=-0.11$, $\mathrm{SEM}=0.24)$. However, a significant difference was noted between the MSos+ and HC groups (mean $=0.92, \mathrm{SEM}=$ $0.39 ; P<0.05$ ). No significant difference was noted between the MS - group and the HC group.

There was no significant effect of group for DKEFS fluency $\left(F_{2,24}=0.2\right.$, NS), CVLT II total learning $\left(F_{2,24}=\right.$ $1.41, \mathrm{NS})$, CVLT II delay recall $\left(F_{2,24}=1.72\right.$, NS), PASAT $\left(F_{2,24}=0.13, \mathrm{NS}\right)$, or arithmetic $\left(F_{2,24}=1.26, \mathrm{NS}\right)$.

\section{DISCUSSION}

Although neuro-ophthalmic syndromes are frequent in MS, to the best of our knowledge, this is the first study to address

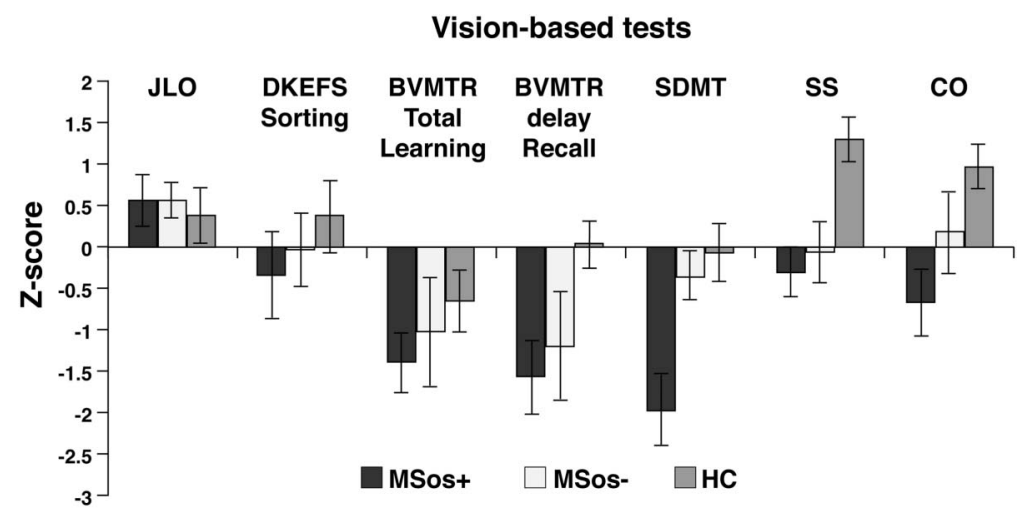

FIG. 1. Mean z score and SEM for each group by vision-based neuropsychological test. BVTMR, Brief Visuospatial Memory Test Revised; CO, Coding Subtest; DKEFS, Delis-Kaplan Executive Function System; HC, healthy controls; JLO, Judgment of Line Orientation test; MSos+, participants with multiple sclerosis with history of neuro-ophthalmic syndrome; MSos-, participants with multiple sclerosis without history of neuro-ophthalmic syndrome; SDMT, Symbol Digit Modalities Test; SEM, standard error of mean; SS, Symbol Search. 


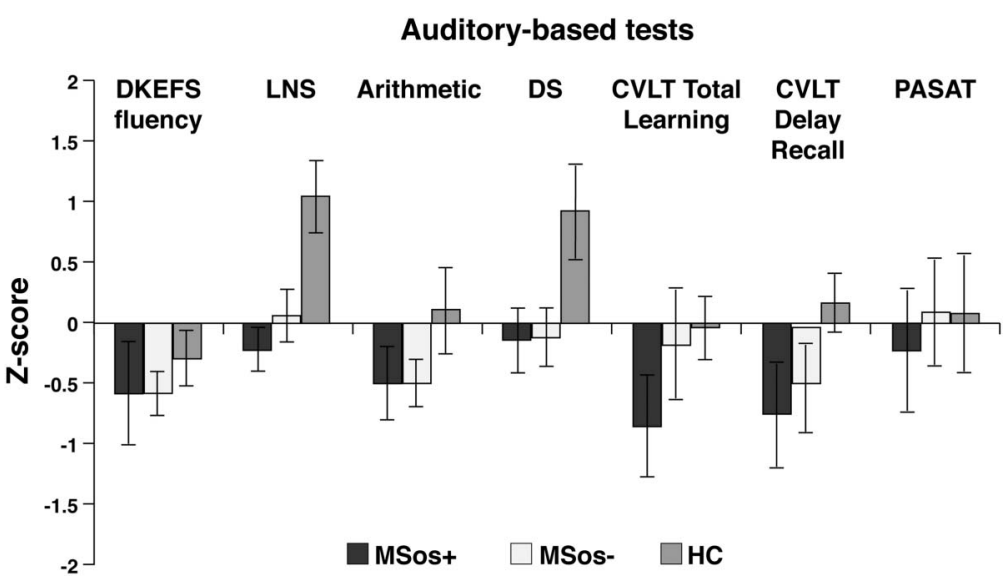

FIG. 2. Mean z score and SEM for each group by auditory-based neuropsychological test. CVLT, California Verbal Learning Test; DKEFS, Delis-Kaplan Executive Function System; DS, Digit Span; HC, health controls; LNS, Letter Number Sequencing; MSos+, participants with multiple sclerosis with history of neuro-ophthalmic syndrome; MSos-, participants with multiple sclerosis without history of neuro-ophthalmic syndromes; PASAT, Paced Auditory Serial Addition Test; SEM, standard error of mean.

the relationship between a self-reported history of neuroophthalmic syndromes and performance on vision- and auditory-based neuropsychological tasks. We showed that a clinical history of neuro-ophthalmic syndromes is associated with decreased performance in visual PS and auditory WM tasks. Poor performance on SDMT was associated with a clinical history of neuro-ophthalmic syndromes. Additionally, participants with a history of neuro-ophthalmic syndromes also performed more poorly on the other 2 PS tasks (SS and CO) compared with the healthy controls. Importantly, no significant differences between the 3 groups were found for the untimed vision-based tests (JLO, BVMT-R learning, and DKEFS Sorting). The only exception was BVMT-R delayed recall, where the MSos+ group did not perform as well as the HC group. Performance on visionbased neuropsychological tests seems to be differentially influenced by the history of neuro-ophthalmic syndromes depending on the time-limited nature of the task. The SDMT is a test widely accepted as sensitive to the detection of cognitive dysfunction in MS (22). It seems that neuro-ophthalmic syndromes are associated with impairments in visual PS despite a preserved ability to processes/perform visual tasks.

Our results suggest that after recovering from a neuroophthalmic syndrome, participants with MS are still able to process visual information but with significant delay. That is, because of deficits on the speed of visual processing, participants will need longer amounts of time to visually process the same quantity of information when compared with healthy controls and participants without a history of neuro-ophthalmic syndromes. Others have reported similar results. Raz et al (3) showed that after an acute episode of optic neuritis, VEP latencies are significantly delayed after 12 months of recovery and are strongly associated with motion perception disturbances. The authors concluded that the temporal aspects of perception are affected because of delays in conduction velocity in the visual pathways.
Neuro-ophthalmic syndromes may lead to impaired function in both the anterior and posterior visual pathways (2) however the ability to process visual information is often preserved. In fact, Snellen visual acuity appears not to be a critical factor in visual PS (PS) in participants with MS, as our groups did not differ in terms of their visual acuity (Table 1). The 3 groups also did not differ in their ability to process visual information, as seen by the performance on JLO, but did differ in their visual PS, as seen on performance on timed visual neuropsychological measures (SDMT, SS and CO).

During an episode of acute optic neuritis, significant reductions occur in amplitude and latency of the VEP. With recovery, amplitude tends to normalize, whereas latencies often remain delayed. A delay in latency seems to be associated with demyelination processes $(23,24)$ and might, in part, explain a reduction in speed-processing visual information. This slowness of visual processing might interfere with performance on SDMT and other measures of visual PS. The SDMT has been found to positively correlate with the average and temporal quadrant RNFL thickness as measured by OCT after controlling for age and number of school years (25). Performance on SDMT reflects the amount of correct responses an individual is able to provide during a 90second interval. If participants with MS need more time to process the visual stimuli, less time will be available to perform the cognitive task, which requires linking numbers to symbols correctly. This is consistent with the Salthouse theory of the limited time mechanisms (26), which postulates that the amount of available time to perform later operations is restricted when a large allocation of resources is involved in the execution of previous operations.

Although no significant differences were found between MS groups on auditory-based neuropsychological tests, differences were noted between both MS groups and the healthy control group. Both MS groups performed significantly worse on LNS relative to the healthy control group. 
However, when examining the standard scores on these tests, it is important to note that the difference between the groups is due to above-average performance of the healthy control group on the auditory processing tasks on which the MS groups performed just below the mean $(\mathrm{z}=-0.025$ to $-0.05)$. WM deficits have been documented in participants with MS (27) with studies documenting that persons with MS performed at significantly lower levels than healthy participants on tasks requiring the manipulation of stored information in WM (28). Yet, other studies failed to document WM deficit in MS (29,30). Studies have directly compared WM and PS abilities in participants with MS and have largely demonstrated a primary deficit in PS rather than WM (31). We have noted similar results in this study. Future research should use both auditory and visual measures of both WM and PS in an attempt to clarify the intricate relationship between these constructs in persons with MS.

We recognize some methodological limitations of our study. First, we used a self-report measure of history of neuroophthalmic syndromes. Additionally, this study documented visual functioning with visual acuity and self-reported abnormalities. Future reports should include more objective documentation including review of medical records, visual fields, contrast acuity, and MNRead. Second, additional measures of disease progression and disability would have been valuable in interpreting the results. In this study, the clinical condition of participants with MS was controlled by using a sample composed solely of participants with relapseremitting course to decrease the potential differences in disease progression. Additionally, the 2 groups of participants with MS were similar in terms of age, education, and months since diagnosis. Future studies should include more comprehensive well-validated disability measures, such as the Expanded Disability Scale to understand its relation with the presence of history of neuro-ophthalmic syndromes and cognitive performance Third, our sample size was small and, fourth, the lack of neuro-imaging data is a limitation of our study. Future research should include magnetic resonance imaging results to quantify disease burden and extent of disease.

\section{STATEMENT OF AUTHORSHIP}

Category 1: a. Conception and design: S. L. Costa, J. Almeida, and O. F. Gonçalves; b. Acquisition of data: S. L. Costa, J. DeLuca, and N. D. Chiaravalloti; c. Analysis and interpretation of data: S. L. Costa, J. Almeida, and O. F. Gonçalves. Category 2: a. Drafting the manuscript: S. L. Costa, J. Almeida, and O. F. Gonçalves; b. Revising it for intellectual content: S. L. Costa, J. Almeida, O. F. Gonçalves, N. D. Chiaravalloti, and J. DeLuca. Category 3: a. Final approval of the completed manuscript: S. L. Costa, J. Almeida, O. F. Gonçalves, N. D. Chiaravalloti, and J. DeLuca.

\section{REFERENCES}

1. Jasse L, Vukusic S, Durand-Dubief F, Vartin C, Piras C, Bernard M, Pelisson D, Confavreux C, Vighetto A, Tilikete C. Persistent visual impairment in multiple sclerosis: prevalence, mechanisms and resulting disability. Mult Scler. 2013;19:1618-1626.

2. Frohman EM, Frohman TC, Zee DS, McColl R, Galetta S. The neuro-ophthalmology of multiple sclerosis. Lancet Neurol. 2005;4:111-121.

3. Raz N, Dotan S, Chokron S, Ben-Hur T, Levin N. Demyelination affects temporal aspects of perception: an optic neuritis study. Ann Neurol. 2012;71:531-538.

4. Tilikete C, Jasse L, Vukusic S, Durand-Dubief F, Vardanian C, Pelisson D, Vighetto A. Persistent ocular motor manifestations and related visual consequences in multiple sclerosis. Ann NY Acad Sci. 2011;1233:327-334.

5. Chiaravalloti ND, DeLuca J. Cognitive impairment in multiple sclerosis. Lancet Neurol. 2008;7:1139-1151.

6. Bruce JM, Bruce AS, Arnett PA. Mild visual acuity disturbances are associated with performance on tests of complex visual attention in MS. J Int Neuropsychol Soc. 2007;13:544-548.

7. Davis AS, Hertza J, Williams RN, Gupta AS, Ohly JG. The influence of corrected visual acuity on visual attention and incidental learning in patients with multiple sclerosis. Appl Neuropsychol. 2009;16:165-168.

8. Feaster HT, Bruce JM. Visual acuity is associated with performance on visual and non-visual neuropsychological tests in multiple sclerosis. Clin Neuropsychol. 2011;25:640-651.

9. White OB, Fielding J. Cognition and eye movements: assessment of cerebral dysfunction. J Neuroophthalmol. 2012;32:266-273.

10. McDonald WI, Compston A, Edan G, Goodkin D, Hartung HP, Lublin FD, McFarland HF, Paty DW, Polman CH, Reingold SC, Sandberg-Wollheim M, Sibley W, Thompson A, van den Noort S, Weinshenker BY, Wolinsky JS. Recommended diagnostic criteria for multiple sclerosis: guidelines from the International Panel on the diagnosis of multiple sclerosis. Ann Neurol. 2001;50:121-127.

11. McCarthy RA, Warrington EK. Cognitive Neuropsychology. A Clinical Introduction. San Diego, CA: Academic Press Inc, 1990.

12. Benedict RH, Fischer JS, Archibald CJ, Arnett PA, Beatty WW, Bobholz J, Chelune GJ, Fisk JD, Langdon DW, Caruso L, Foley F, LaRocca NG, Vowels L, Weinstein A, DeLuca J, Rao SM, Munschauer F. Minimal neuropsychological assessment of MS patients: a consensus approach. Clin Neuropsychol. 2002;16:381-397.

13. Delis DC, Kaplan E, Kramer JH. Delis-Kaplan Executive Funciton System. San Antonio, TX: Psychological Corporation, 2001.

14. Benton AL, Sivan AB, Hamsher K, Varney NR, Spreen O. Contributions to Neuropsychological Assessment, 2nd edition. New York, NY: Oxford University Press, 1994.

15. Delis DC, Kramer JH, Kaplan E, Ober BA. California Verbal Learning Test Manuel: 2nd Edition, Adult Version. San Antonio: TX: Psychological Corporation, 2000.

16. Benedict RH. Brief Visuospatial Memory Test-Revised: Professional Manual. Odessa, Ukraine: Psychological Assessment Resources, Inc, 1997.

17. Smith A. Symbol Digit Modalities Test: Manual. Los Angeles, CA: Western Psychological Services, 1982.

18. Rao SM, Leo GJ, Bernardin L, Unverzagt F. Cognitive dysfunction in multiple sclerosis. I. Frequency, patterns, and prediction. Neurology. 1991;41:685-691.

19. Parmenter BA, Testa SM, Schretlen DJ, Weinstock-Guttman B Benedict RH. The utility of regression-based norms in interpreting the minimal assessment of cognitive function in multiple sclerosis (MACFIMS). J Int Neuropsychol Soc. 2010;16:6-16.

20. Wechsler D. Wechsler Adult Intelligence Scale-3rd Edition. San Antonio, TX: Psychological Corporation, 1997.

21. Wechsler D. Wechsler Adult Intelligence Scale-4th Edition. San Antonio, TX: Pearson, 2008.

22. Benedict RH, Duquin JA, Jurgensen S, Rudick RA, Feitcher J, Munschauer FE, Panzara MA, Weinstock-Guttman B. Repeated assessment of neuropsychological deficits in multiple sclerosis using the Symbol Digit Modalities Test and the MS 
Neuropsychological Screening Questionnaire. Mult Scler. 2008;14:940-946.

23. Almarcegui C, Dolz I, Pueyo V, Garcia E, Fernandez FJ, Martin J, Ara JR, Honrubia F. Correlation between functional and structural assessments of the optic nerve and retina in multiple sclerosis patients. Neurophysiol Clin. 2010;40:129-135.

24. Burton EV, Greenberg BM, Frohman EM. Optic neuritis: a mechanistic view. Pathophysiology. 2011;18:81-92.

25. Toledo J, Sepulcre J, Salinas-Alaman A, Garcia-Layana A, MurieFernandez M, Bejarano B, Villoslada P. Retinal nerve fiber layer atrophy is associated with physical and cognitive disability in multiple sclerosis. Mult Scler. 2008;14:906-912.

26. Salthouse TA. The processing-speed theory of adult age differences in cognition. Psychol Rev. 1996;103:403-428.

27. Chiaravalloti N, Hillary F, Ricker J, Christodoulou C, Kalnin A, Liu WC, Steffener J, DeLuca J. Cerebral activation patterns during working memory performance in multiple sclerosis using FMRI. J Clin Exp Neuropsychol. 2005;27:33-54.

28. Forn C, Belenguer A, Parcet-lbars MA, Avila C. Informationprocessing speed is the primary deficit underlying the poor performance of multiple sclerosis patients in the Paced Auditory Serial Addition Test (PASAT). J Clin Exp Neuropsychol. 2008;30:789-796.

29. Olivares T, Nieto A, Sanchez MP, Wollmann T, Hernandez MA, Barroso J. Pattern of neuropsychological impairment in the early phase of relapsing-remitting multiple sclerosis. Mult Scler. 2005;11:191-197.

30. Schulz D, Kopp B, Kunkel A, Faiss JH. Cognition in the early stage of multiple sclerosis. J Neurol. 2006;25:1002-1010.

31. Kalmar JH, Bryant D, Tulsky DS, DeLuca J. Information processing deficits in multiple sclerosis: does choice of screening instrument make a difference? Rehabil Psychol. 2004;49:213-218. 